\title{
Wavelet-like selective representations of multidirectional structures: a mammography case
}

\author{
Magdalena Jasionowska $^{1}\left[\right.$ Artur Przelaskowski $^{1}$
}

Received: 23 January 2017 / Accepted: 11 March 2018 / Published online: 17 March 2018

(c) The Author(s) 2018

\begin{abstract}
The subject of this paper is selective representation of informative texture directionality in sparse domain of four verified multiscale transforms: contourlets, curvelets, tensor and complex wavelets. Directionality of linear or piecewise linear structures is a fundamental property in recognition of anatomical structures, i.e. separating the brain regions, directional characteristics of small coronary arteries. Another important example is spicule extraction in mammograms which is based on directional analysis of malignant spiculated lesions. The originality of the proposed experiments lies in optimization of multiscale wavelet-like representations to differentiate multidirectional structures of architectural distortions and spiculated masses in low-contrast noisy mammograms. For that purpose, the applied method consists in proposed phantom-based normalization and defined assessment criteria of directional information activity. The directional activity including both measures of angular resolution and angular selectivity was determined relative to increased background density and noise simulating reduced perceptibility of the analyzed objects. A numerically modeled phantom of multidirectional linear structures was used to assess subjectively and objectively the efficiency of nonlinear approximation for radially diverging spicule-like structures. Size and distribution of the structures simulates essential nature of spiculated signs of breast cancer. Basing on the experimental results, the complex wavelet domain was concluded to be the most effective tool to uniquely represent relevant information in the form of multidirectional piecewise linear structures in low-contrast noisy mammograms.
\end{abstract}

Keywords Multiscale transform domains $\cdot$ Angular selectivity $\cdot$ Angular resolution $\cdot$ Mammographic spicules representation

\section{Introduction}

Although limited, mammography proves useful as a screening tool for early detection of breast cancer. The effectiveness of diagnosis of some radiological signs of cancer is moderate, especially in case of architectural distortions (ADs) and subtle spiculated masses (SMs) [1]. These pathologies are commonly misdiagnosed even by the most experienced radiologists. Hence, computer-aided diagnosis (CAD) systems have been undergoing improvements for years due to significantly lower efficiency in ADs detection compared to high-efficiency

Magdalena Jasionowska

jasionowskam@mini.pw.edu.pl

Artur Przelaskowski

arturp@mini.pw.edu.pl

1 The Faculty of Mathematics and Information Science, Warsaw University of Technology, Koszykowa 75, 00-662 Warsaw, Poland detection of both masses and calcifications. 56\% sensitivity of detection was achieved for subtle SMs, whereas typical SMs were computer-detected with $86 \%$ sensitivity at 0.24 false positives per image (FPI) [2]. Simultaneously, two commercial CAD systems (R2 Image Checker [3] and Second Look CADx [4]) detect ADs with low sensitivity being less than $40 \%$ at approx. 1.0 FPI [2]. Moreover, the detection efficiency of pathological findings with the use of CAD systems decreases with increasing breast tissue density [5]. Both SMs and ADs are commonly misdiagnosed even by the most experienced radiologists in screening mammography. This is a problem in early detection of pathologies and, consequently, in full recovery from breast cancer. Spiculated findings in mammograms are manifested by radiating straight line structures in various directions from the hypothetical center. The lack of stable patterns of both normal and abnormal breast tissues tends to have an adverse effect on automatic detection of subtle spiculated lesions. However, local directional distribution of spiculated structures tends to be a dominant distinguishing factor for 
both ADs and SMs in computer-aided detection methods, based on the review of the literature.

Instead of a statistical texture analysis in the image domain, an analysis of spicule distribution in scalable transform domains was proposed, including wavelets [6] and their extensions to 2D kernels, complex domains and optimized filter set-based implementations [7-11]. The scalability of transforms makes it possible to obtain sparse representation which is more flexible for studying the dependence of the specific features of the spiculated pathologies (ADs and SMs) at different scales, as well as for determination of scale-invariant numerical descriptors. Consequently, several properties of normalized sparse representation are obtainable, i.e. signal energy compaction against energy dissipation of noise, shift (rotation) invariance, a sufficiently high directional selectivity, and low computational complexity. Therefore, the signal-adjusted tensor wavelets [6] and the extensions with non-separable 2D kernels: curvelets [7, 8], contourlets [9], and complex wavelets [10,11] were considered due to the desired properties of content-oriented image representation. The selected set of transform domains reflects simplicity, flexibility of design, and popularity of tensor wavelets in medical imaging applications. Curvelets and contourlets increase description sparsity of smooth curved lines and multidirectional straight line structures, whereas complex wavelets assure shift invariance and an additional directional analysis in a phase domain. Nonlinear approximation of multidirectional structures was optimized in signal sparse domains. Precise determination of mammographic spicules can frequently be problematic due to their subtlety. Consequently, the radiologists' assessments of these barely visible pathologies in mammograms are frequently unrepeatable [12].

\section{Materials and methods}

The main goal of the study is directional characteristics of local piecewise linear structures in image, which accurately manifest the mammographic signs of malignancy with the use of the most appropriate multiscale transform domain. For this purpose, the initial experiments were conducted with the use of the designed spicule phantom with close-lying linear structures of limited length and with a wide angular spectrum. Two criteria: angular selectivity and angular resolution, were proposed for the determination of the local multidirectional activity of piecewise linear objects, both related to the number of detected structures with at least half of their length in reconstructed images. The experiments, based on the multidirectional activity measures, were conducted with the use of a spicule phantom model with different noise levels and various density characteristics of linear structures distribution. Subsequently, the results of the phantom model-based optimization were verified on a test set of real mammograms.
The novelty of the paper method lies in all of the four components: spicule phantom model design, a proposal of directional activity measures, verification of the utility of wavelet-like transforms, and a proof of concept for the recognition of both ADs and SMs in real mammograms.

\subsection{Directional activity measures}

Two criteria of directional activity: angular resolution and angular selectivity, were proposed to identify the image transformation suitable for the effective (i.e. selective and discriminative) representation of specific orientation activity of piecewise linear structures. Hence for this purpose, angular resolution was specified as the minimum angular separation of two radiating lines, while the separation is perceived or computed along at least half of their length. The minimum angle of separation is modeled by a phantom of close-lying lines originating from a relatively small center with a controlled angular distance. Simultaneously, angular selectivity (also called directional resolution) was defined as the maximum number of lines or piecewise lines originating from a small center which propagate in different directions of a full angle and are perceptually or computationally separable along at least half of their length.

\subsection{Spicule phantom model}

Not only multiscale directional analysis, but also a shift (including rotation) invariance of image transforms, low transformdomain redundancy and low computational complexity play a role in determination of the ability to represent multidirectional structures. Selection of the optimal image transforms is based on as close as possible preservation of object orientations in transform domains in relation to original images. Considering those properties, the bases or frames of tensor wavelets, complex wavelets, contourlets and curvelets were selected to be experimentally verified, based on a spicule phantom model which contains object (line) value of 1 and slightly Gaussian blurred lines of approx. 9-pixel in width and 90-pixel in length which are estimated averaged sizes of spicules according to our previous study [13]. The angle range of line distribution was assumed as: $0^{\circ}-360^{\circ}$ and different center size of line convergence was adopted: the sparse phantom (Fig. 1a), the mediumdensity phantom (Fig. 1b), and the dense phantom (Fig. 1c). Realistic conditions for object-background relationship, based on [14], were generated with the value change of two parameters: the global contrast level and the noise level. Global contrast level $\left(C_{\text {glob }}\right)$ was defined as follows:

$$
C_{\text {glob }}=\frac{\operatorname{mean}\left[P h_{o b j}(i, j)\right]-\operatorname{mean}\left[P h_{\text {backgr }}(i, j)\right]}{\max \left[\max _{i, j} P h_{o b j}(i, j), \max _{i, j} P h_{\text {backgr }}(i, j)\right]}
$$


(a)

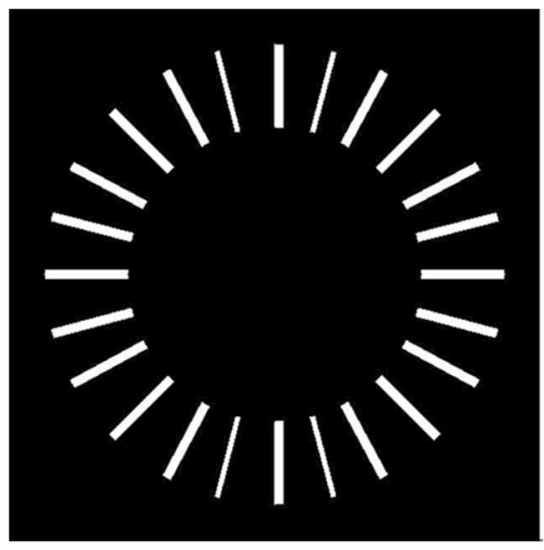

(b)

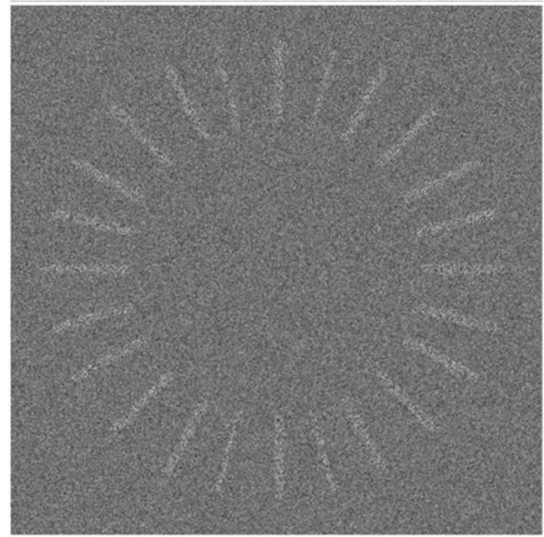

(c)

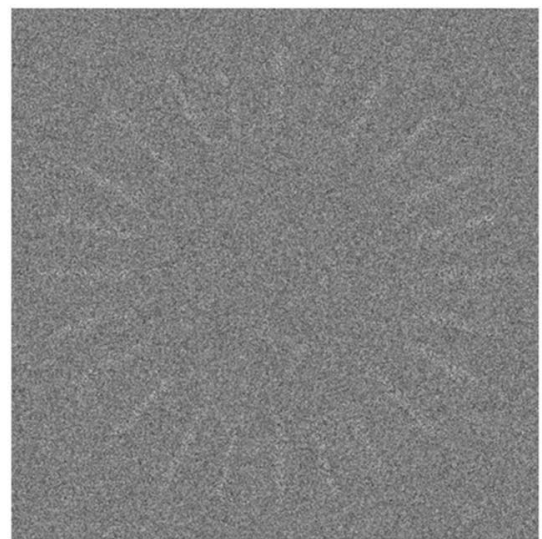

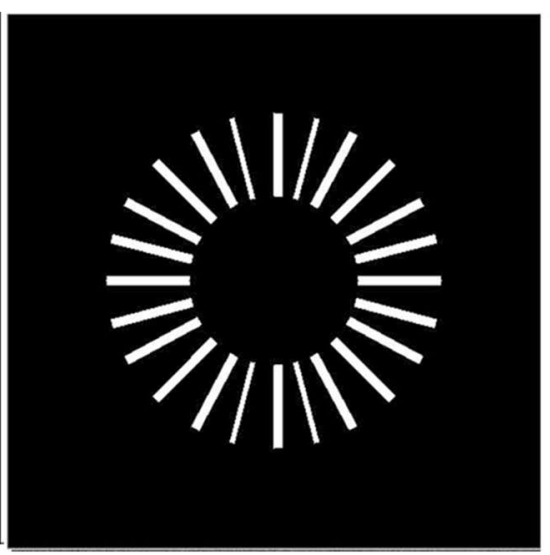
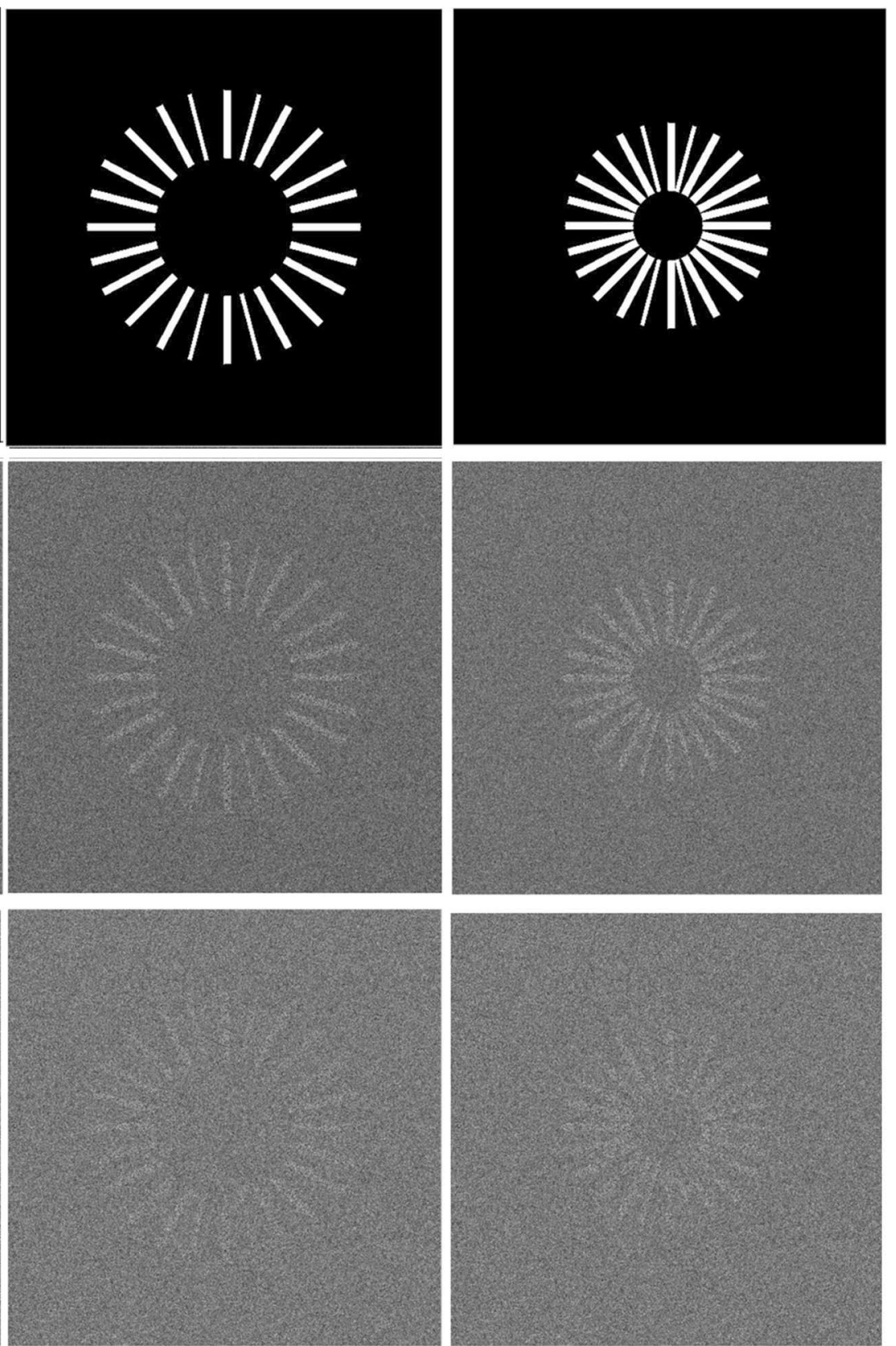

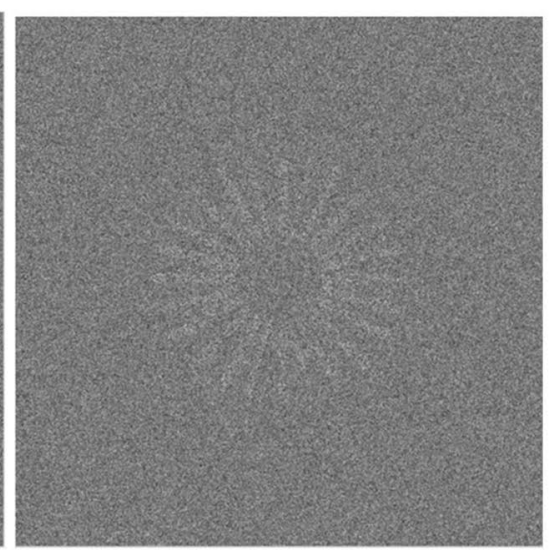

Fig. 1 a Various forms of spicule phantom model with varying spicule density levels: sparse, medium-density, dense phantom of linear structures radiating from the convergence center of different size (left to right). $\mathbf{b}$ and $\mathbf{c}$ Various forms of spicule phantom model with multiplicative Gaussian noise. Noise level, being background density level, was defined on the basis of varying background pixel-value equal b 0.5 , c 0.7 , resulting in $C_{\text {glob }}=7.5$ and 4.5 , PSNR $=2.78$ and 1.46 , respectively where mean $\left[P h_{o b j}(i, j)\right]$ is the mean pixel-value for the objects, mean $\left[P h_{o b j}(i, j)\right]$ being the mean pixel-value for the spicule phantom background. For the phantom model with background pixel-value equal $0, C_{\text {glob }}$ was set to approx. $76 \%$ and decreased with the step size of approx. $1.5 \%$, caused by increasing background pixel-value in the range of 0-0.9.

Additionally, signal-dependent and zero-mean Gaussian noise $\varepsilon$ with standard deviation $\sigma=1$ was added to the phantom model [14]. The phantom model with various noise level $P h_{\varepsilon}(i, j)$ (Fig. 1b, c) was calculated as follows:

$P h_{\varepsilon}(i, j)=P h(i, j)+\varepsilon \sqrt{P h(i, j)}$

where $P h(i, j)$ is a spicule phantom model with structures which are diverse in terms of both the propagation density and the object-background relation. $C_{\text {glob }}$ reached $16 \%$ for the phantom model with background pixel-value equal 0 and 
gradually decreased by $1.5 \%$ with increasing background pixel-value.

In this approach, the peak signal-to-noise ratio (PSNR) was defined as follows:

$$
\text { PSNR }=10 \log \frac{\left(\max _{i, j} P h(i, j)\right)^{2}}{\frac{1}{N \times M} \sum_{1}^{N} \sum_{1}^{M}\left(P h(i, j)-P h_{\varepsilon}(i, j)\right)^{2}}
$$

where $P h(i, j)$ is the appropriate spicule phantom of the selected $C_{\text {glob }}$, with $P h_{\varepsilon}(i, j)$ being the phantom $P h(i, j)$ with multiplicative noise $\varepsilon$ and $N \times M$ being the size of images, i.e. $512 \times 512$. PSNR decreased from near $12-0.5 \mathrm{~dB}$.

With such assumptions, both different size of pathological findings and the diversified density of breast tissue were simulated with the use of increasing background pixel-value and noise level in relation to the analyzed objects.

\subsection{Directional activity of multiscale wavelet-like domains}

It is challenging to objectively characterize and reliably compare both angular resolution and angular selectivity of competitive multiscale domains. It is probably caused by a number of diverse factors characterizing discrete transforms, frequently dependent on others, i.e. variable redundancy of transform domains, energy compaction, susceptibility to aliasing, limited invariance of structure representation, variable numbers of directional subbands, homogeneity of angular resolution, etc. Therefore, an initial experiment, based on noisy spicule phantom model, was conducted to assess the directional activity of multiscale transform domains. For this purpose, the MATLAB toolboxes of four selected image transform domains were used and both the type of filters and the number of scales and appropriate subbands of decomposition were adjusted to effectively extract the modeled spicules from the various background. Three types of image background were presented in this paper as a simulation of breast tissue: fat tissue, fat-glandular tissue and glandular tissue, respectively. As a result, there were computed:

- 2-dimensional tensor wavelet transform [6] for three scales and three subbands with low-pass orthonormal quadrature mirror filters (QMF), called Battle-Lemarie, which generate spline orthogonal wavelet basis, the degree of the spline being 5 [15],

- contourlet transform [9] for five scales, where the vector of numbers of directional filter bank decomposition levels at each of the five pyramidal levels (from coarse to fine scale) was determined as follows: $\left[2^{0}, 2^{0}, 2^{0}, 2^{3}, 2^{4}\right]$, and the 9-7 filter was used for the pyramidal decomposi- tion step, whereas the pkva filter was used for the directional decomposition step [16];

- complex-valued curvelet transform [7] for four scales, including the coarsest wavelet level, and three directional scales, where the coarsest scale was divided into eight angular ranges [7];

- complex wavelet transform with six levels of decomposition, near-symmetric $(13,19)$-tap filters as biorthogonal filters and $Q$-Shift orthogonal $(10,10)$-tap filters $[10,11]$; six complex highpass subimages for each of all levels were generated as the result of the transformation.

The main aim of the present paper was to verify the ability of wavelet-like transforms to diversify multidirectional structures. Based on the four decomposition settings and the two proposed measures of directional activity, the number of noticeable model-structures in the reconstructed images corresponds to the angular selectivity, whereas the analysis of degree of structure compression indicates the angular resolution.

\section{Results and discussion}

Our research was divided into two parts. Firstly, an attempt was made to verify the repeatability of linear structure orientations in the reconstructed images with the use of tensor wavelets, contourlets, curvelets, and complex wavelets. Based on the experiments with the spicule phantom model with no added noise (Fig. 1a), but with nonlinear approximation of transformation coefficients (with the criterion of its absolute value order) it may be noticed that most of the analyzed transformations distort orientations of spicule phantom model (Figs. 2, 3). These distortions are negligible and diagnostically unimportant merely in the case of the complex wavelet domains (the least significant deviations from the original structure directions). Additionally, the most accurate representation of structures was achieved in the complex wavelet domain. Accurate representation was construed as a smooth structure reconstruction, maintaining precise orientation of the analyzed structures (Figs. 2, 3 ). Continuous nature of the multidirectional piecewise linear structures was best preserved in the complex wavelet domain. Moreover, the angular selectivity of the analyzed transforms varies depending on the propagation density of the structures. The higher the propagation density was, the lower the angular selectivity was in all of the analyzed domains.

Secondly, an attempt was made to determine the directional activity, based on angular resolution and selectivity of tensor wavelets, contourlets, curvelets and complex wavelets relative to sparse representation of objects in the spicule phantom model. Sparse representation is 
(a)
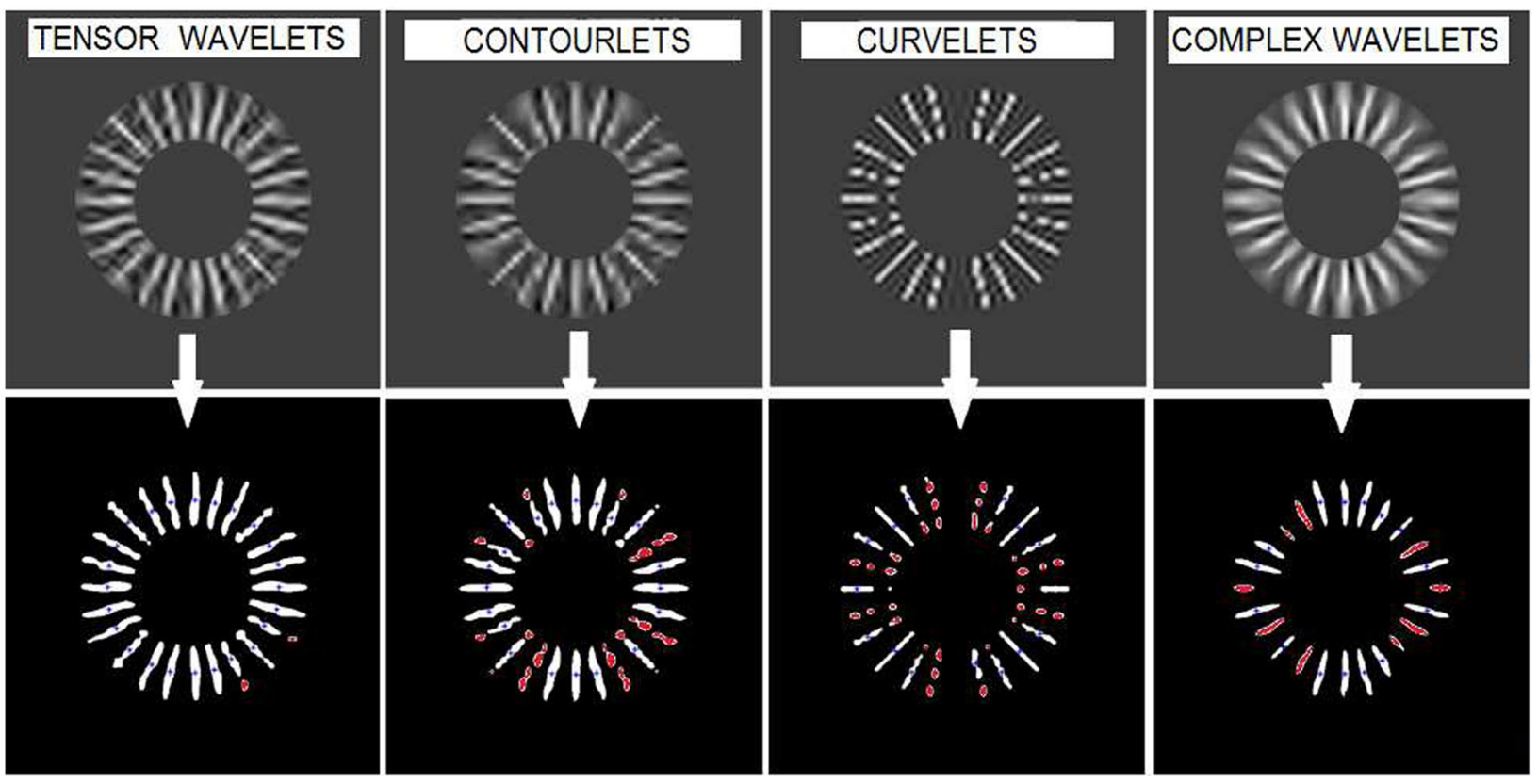

Fig. 2 a The reconstructed images based on $n=200$ : tensor wavelet, contourlet, curvelet, and complex wavelet coefficients of the highest amplitude values for the medium-dense spicule phantom model, $\mathbf{b}$ the results of detection with the use of binarization of the reconstructed

images with two thresholds: $t_{b}=0.5$, and $t_{d s}=0.5 l_{o b j}, l_{o b j}$-linear structure length. The unidentified objects were color-marked (color figure online)

(a)

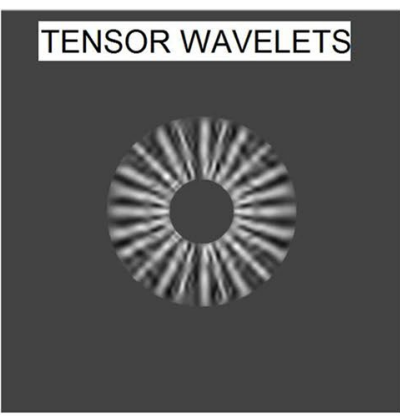

(b)
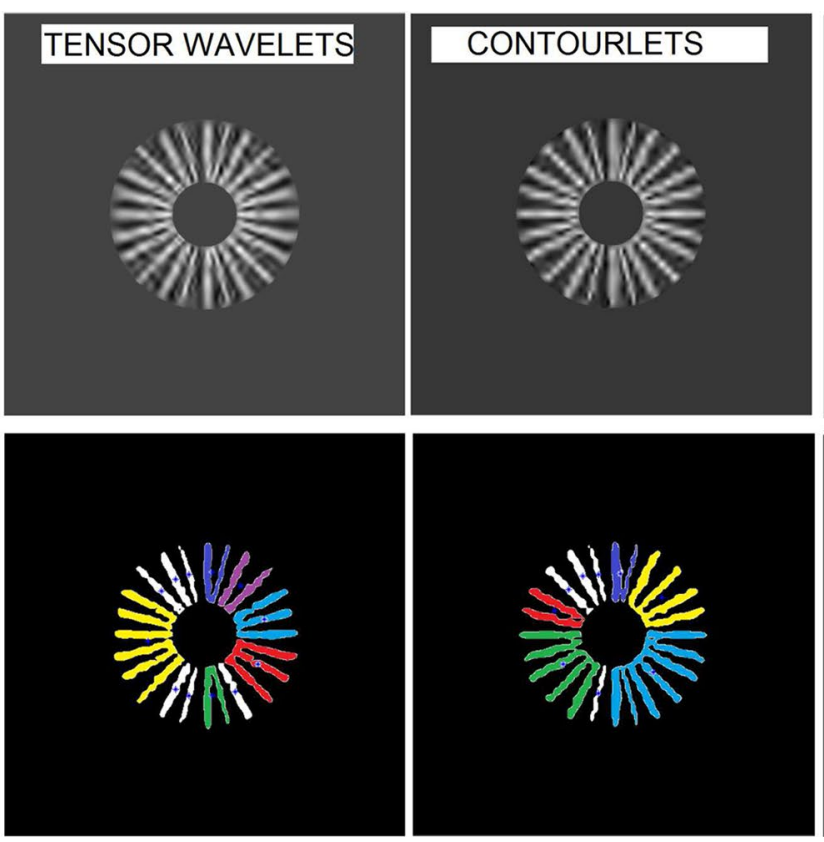
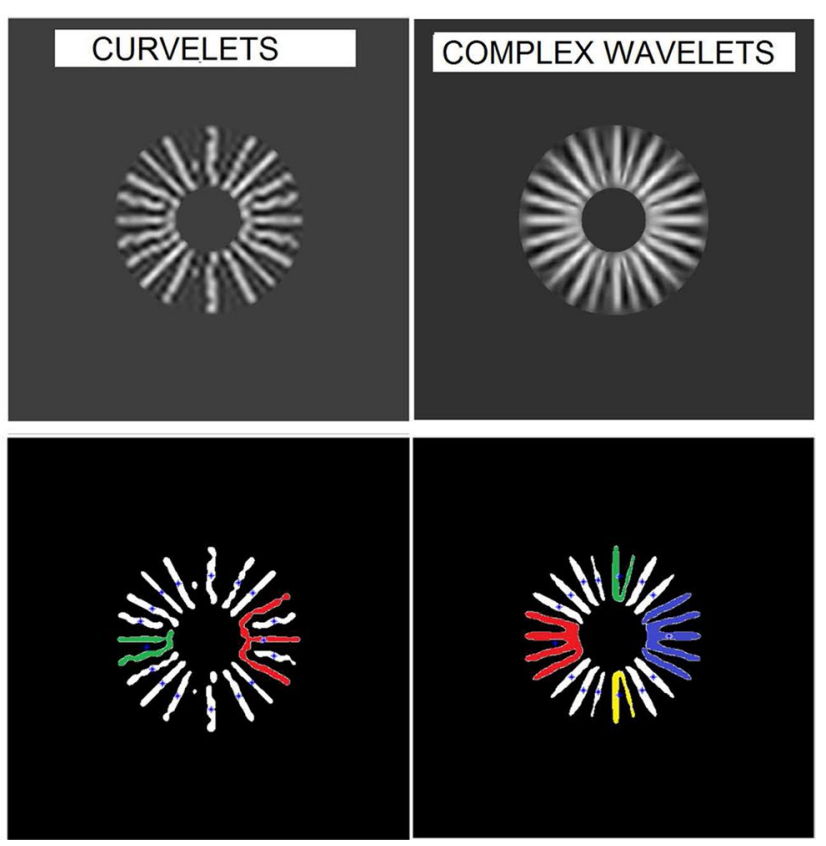

Fig. 3 a The reconstructed images based on $n=200$ : tensor wavelet, contourlet, curvelet, complex wavelet coefficients of the highest amplitude values for the dense spicule phantom model, $\mathbf{b}$ the detection results with the use of binarization of the reconstructed images

a consequence of the coefficient reduction with the use of approximation techniques. The reconstruction images based on the significant decomposition coefficients are with two thresholds: $t_{b}=0.5$, and $t_{d s}=0.5 \mathrm{lobj}, l_{o b j}$-linear structure length. The several structures detected as one object were colormarked (color figure online)

applied to determine the ability of the selected multiscale transforms to represent multidirectional structures. The minimum number of decomposition coefficients, sufficient 
to perceptually and computationally distinguish all linear structures in the phantom along at least half their length, depends on three factors: the used transform domain, the density of radiating linear structures, and the surrounding background (tissue density).

Sparse approximation of coefficients tends to be a useful way to enhance linear structures, primarily in two of the four analyzed domains, i.e. the curvelet and the complex wavelet domain (Fig. 4). In both the tensor wavelet and the contourlet domains, the process of separation and differentiation of linear structures of various orientations becomes increasingly difficult when these objects are progressively compressed, whereas the detection of phantom objects tends to be more effective in the curvelet and complex wavelet domains.

The results of the initial experiments with the use of spicule phantom model with the added noise (Fig. 4) can be considered as another interesting aspect of multidirectional lines recognition. In this case, it is complex wavelets rather than curvelets that have a higher potential to detect multidirectional structures. Using curvelets, single linear structures may be detected as several smaller structures due to the lack of continuity across the analyzed linear structures. Hence, the number of detected objects in the reconstructed images may be distorted.
Fig. 5 The results of sparse nonlinear approximation in the tensor wavelet, contourlet, curvelet and complex wavelet domains for mammograms [17] with architectural distortions (a, b) and normal breast tissue (c). The reconstructed images based on $n=300$ and 600 coefficients of the highest amplitude values

Moreover, the higher was the observed background density level, the higher was the number of significant coefficients necessary for a complete signal description. Furthermore, the effective sparse approximation of transform coefficients depends on the type of image transformation rather than the density of piecewise linear structures in the curvelet and complex wavelet domains. Based on the experiment in the complex wavelet domain, it appears that the significant coefficients were mainly concentrated in the regions with the objects of interests, and subsequently around diagnostically irrelevant information of image content. Informative energy accumulation of image content was differentiated more fuzzily in other domains in view of the absolute value of coefficients. Hence, it was more possible to determine the threshold between the informative and uninformative image content in the complex wavelet domain than in other domains (Fig. 4-complex wavelets).

This observation was confirmed in the conducted tests with real mammograms, especially for a small number of
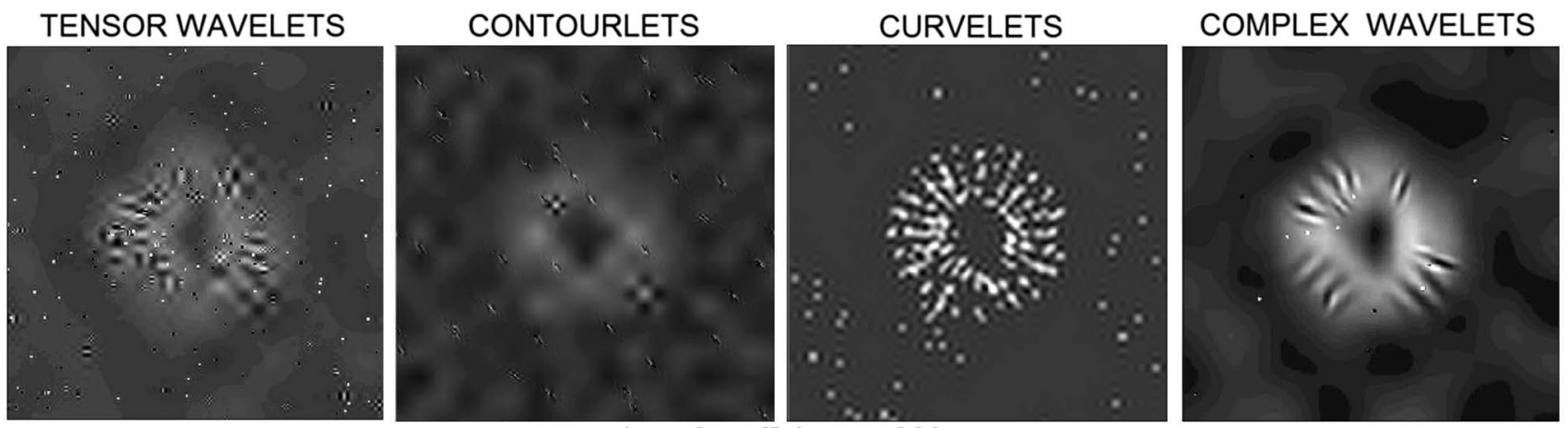

number of coefficients $=300$
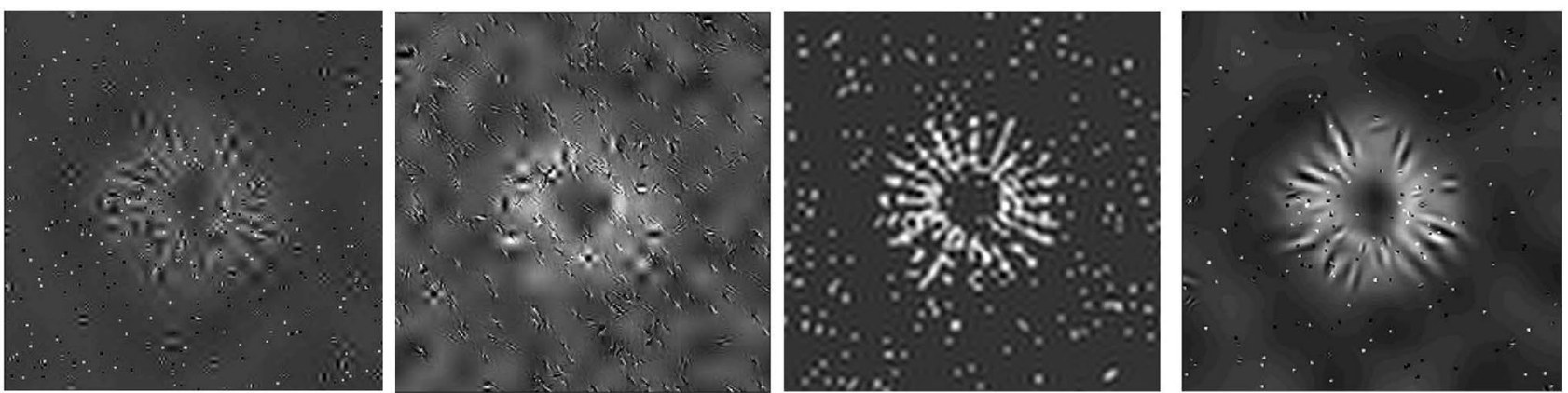

number of coefficients $=600$

Fig. 4 The results of sparse nonlinear approximation in the tensor wavelet, contourlet, curvelet and complex wavelet domains for the dense phantom of high background density $C_{\text {glob }}=4.5, \operatorname{PSNR}=1.46$
(Fig. 1c-right). The reconstructed images based on $n=300$ and 600 coefficients of the highest amplitude values 


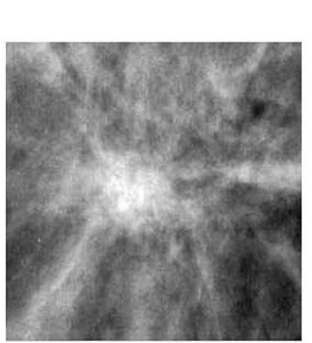

(a)
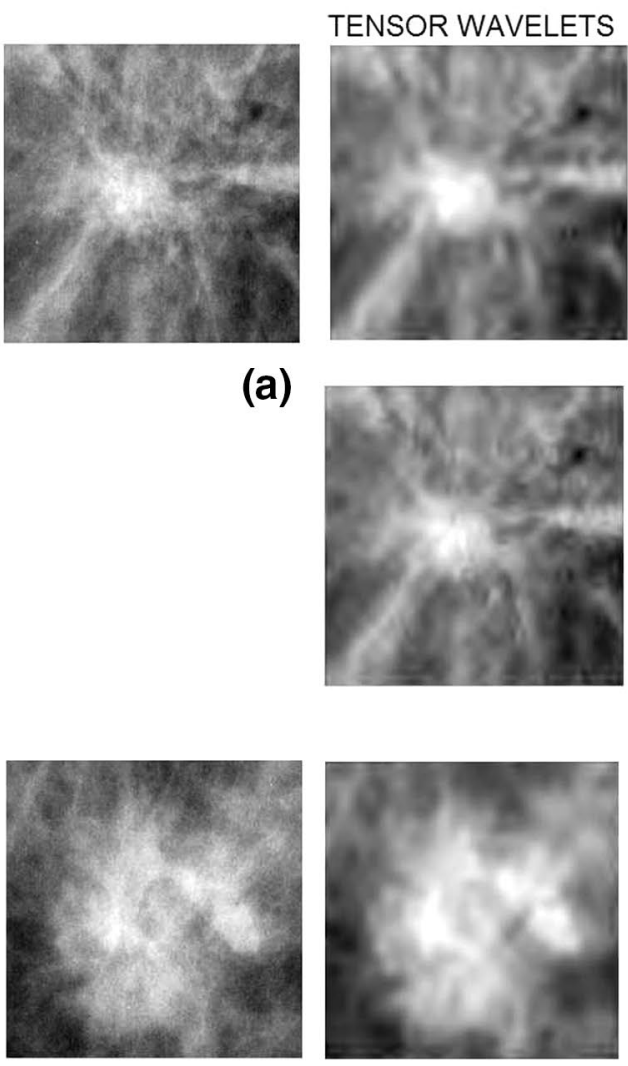

(b)
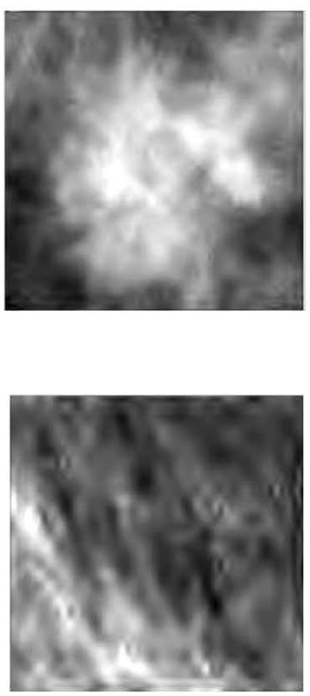

(c)
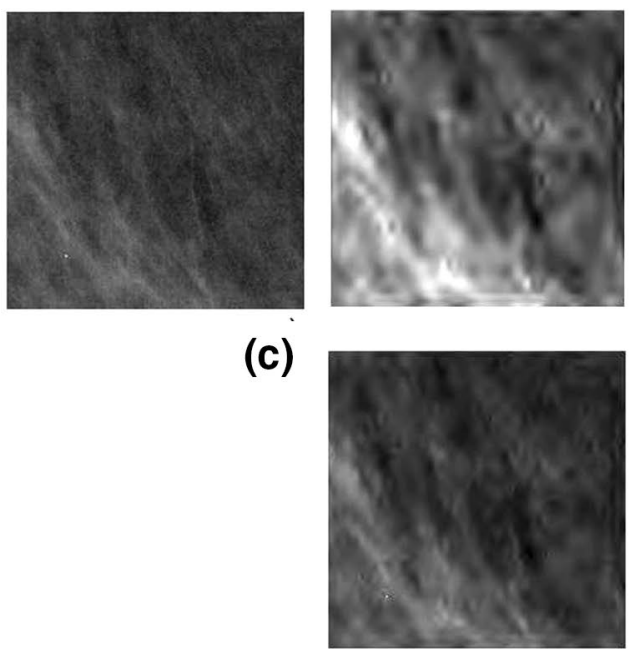

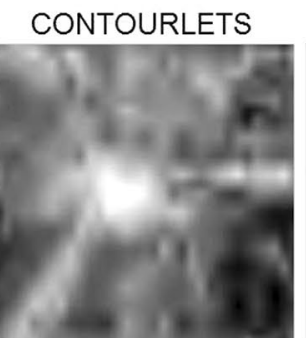

CURVELETS

COMPLEX WAVELETS

number of coefficients $=300$
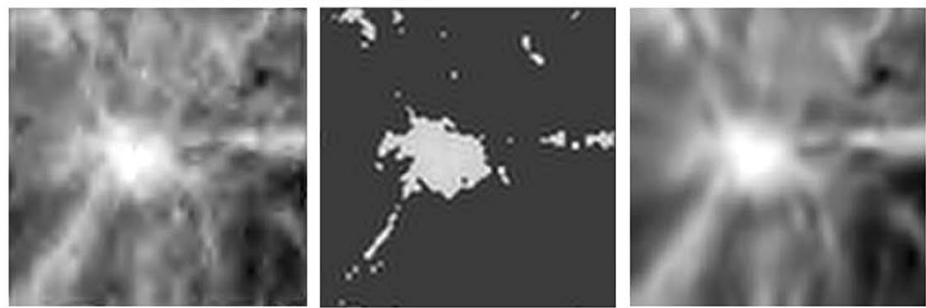

number of coefficients $=600$
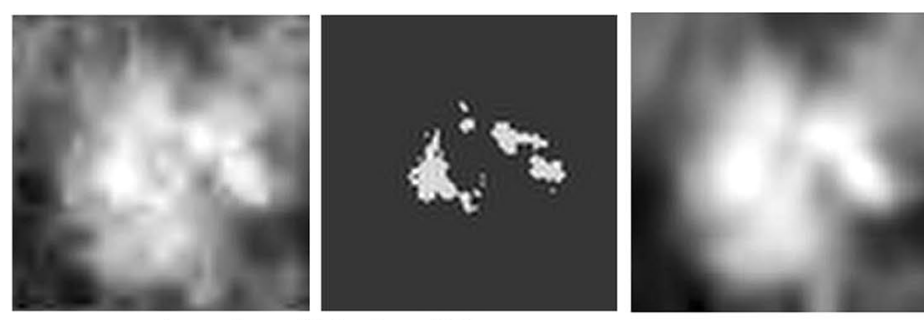

number of coefficients $=300$
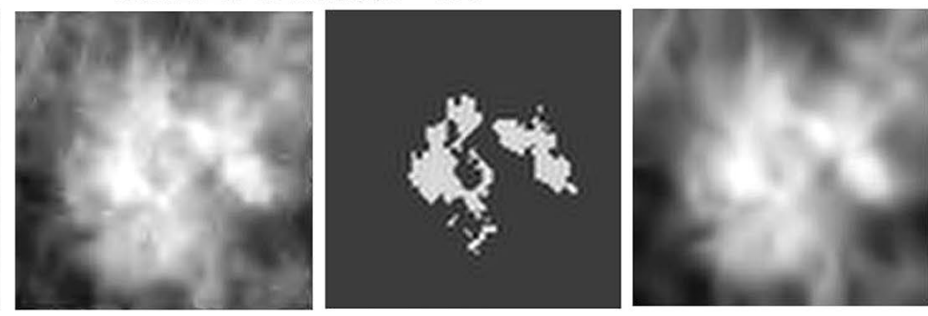

number of coefficients $=600$
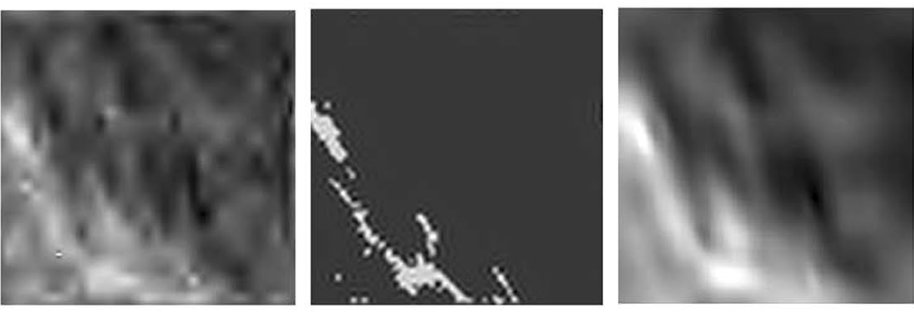

number of coefficients $=300$
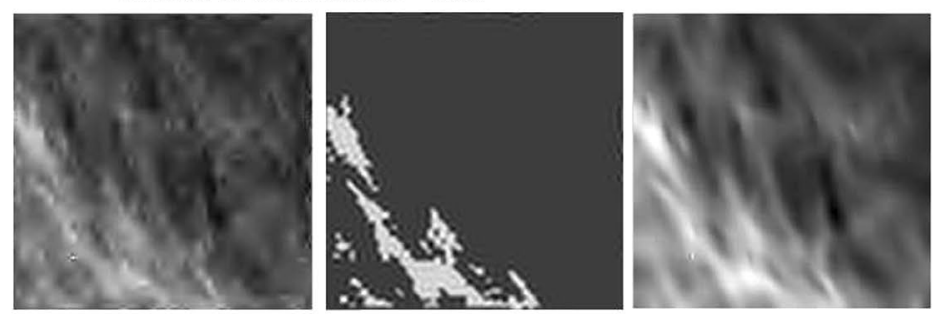

number of coefficients $=600$ 
approximation coefficients (Fig. 5-complex wavelets). Sparse representation of spicules in the complex wavelet domain tends to result in a dominant angularity distribution of linear spicules. The informative image content becomes less pronounced with an increased number of approximation coefficients due to a more accurate restoration of both noise and uninformative structures, together with irregular details of spicules, making them similar to other structures. Spicule discrimination appears less distinctive in case of the other domains (Fig. 5-tensor wavelets and contourlets). The superiority of complex wavelets in spicule representation, fairly noticeable in subjective assessment, was confirmed by the proposed objective assessment measure of spicule representation (Figs. 6, 7), defined as the product of correlation (CORR) and informative measure of correlation (IIMCl).

Correlation is defined as the marginal-probability over rows $\left(p_{x}\right)$ and columns $\left(p_{y}\right)$ of the normalized co-occurrence matrix $(p(i, j))$ from image:

$\mathrm{CORR}=\frac{\sum_{i} \sum_{j}(i j) p(i, j)-\mu_{x} \mu_{y}}{\sigma_{x} \sigma_{y}}$

where $\mu_{x}, \mu_{y}, \sigma_{x}, \sigma_{y}$-the means and standard deviations of $p_{x}$ and $p_{y}, i, j \in\left[0, I_{\max }\right], I_{\max }$-image intensity [18].

Informative measure of correlation is defined as follows:

$\mathrm{IMC}=\frac{\max (H X, H Y)}{H X Y-H X Y 1}$

where

$H X Y=-\sum_{i} \sum_{j} p(i, j) \log (p(i, j))$

$H X Y 1=-\sum_{i} \sum_{j} p(i, j) \log p_{x}(i), p_{y}(j)$

and $H X, H Y$-entropies of $p_{x}$ and $p_{y}[18]$.

The CORR $*$ IMC measure concentrates on the calculation of correlation of restored neighbor pixels with averaged contexts. Higher correlations of restored data (due to the nonlinear approximation with significantly reduced numbers of the applied transform coefficients) mean above all

- denoising and blurring of details,

- sparse representation for smooth structures which are informative image content,

- smoothness versus coarseness of the reconstructed structures,

- continuous representation of the holistic structure core in the compact form of the approximated structures without splitting or irregularity.

In addition, lower entropy of the joint probability of the normalized co-occurrence matrix relative to the entropy, which is calculated for marginal probabilities for rows and columns, means an increase in compacted data dependence in a full spatial context of multidirectional analysis in the averaged $2 \mathrm{D}$ context.

Both subjective (Figs. 4, 5) and objective (Figs. 6, 7) assessments confirm practical usefulness of complex wavelets in the extraction of different oriented spicules in noisy real mammograms. To sum up, it can be assumed that the complex wavelet transform is quite an effective tool for analyzing and extracting the subtle piecewise linear structures in images due to shift invariant, appropriate angular resolution and selection and, consequently, extraction of diagnostically relevant image content.

\section{Conclusions}

This paper attempts to show that sparse representation of texture directionality in the transform domain is a desirable tool to extract directionality of piecewise linear structures (here, to extract mammographic spicules). However, precise determination of angular resolution and angular selectivity of four selected multiscale transformations is an interesting, yet unstudied issue in mammogram preprocessing due to
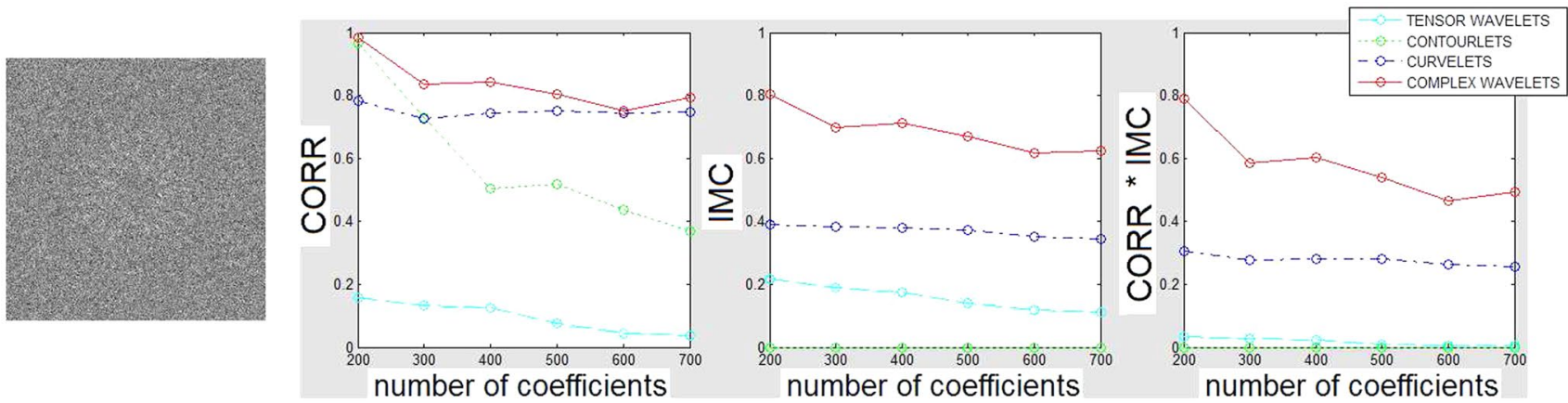

Fig. 6 The proposed objective assessment measures of spicule representation for the dense phantom of high density $C_{\text {glob }}=4.5$, PSNR $=1.46$ (Fig. 1c-right). The dependence of CORR, IMC and CORR $*$ IMC on the number of approximation coefficients 


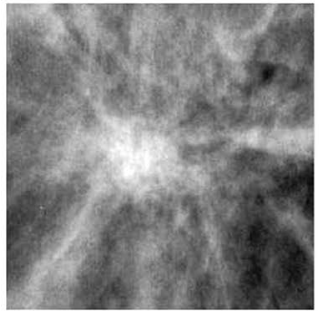

(a)

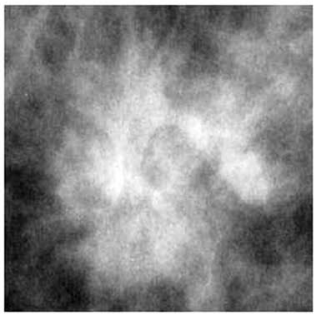

(b)

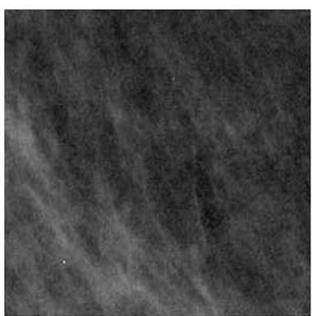

(c)
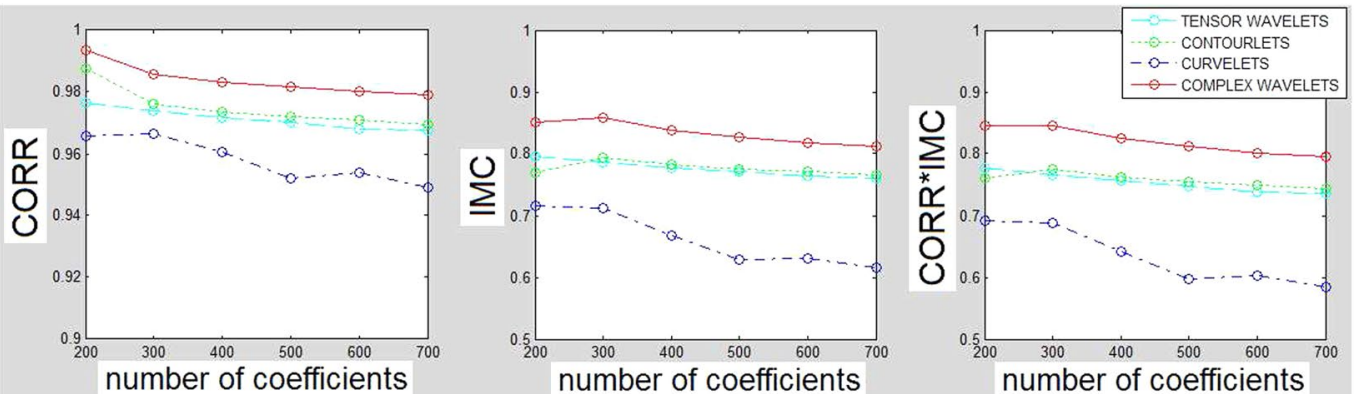

number of coefficients
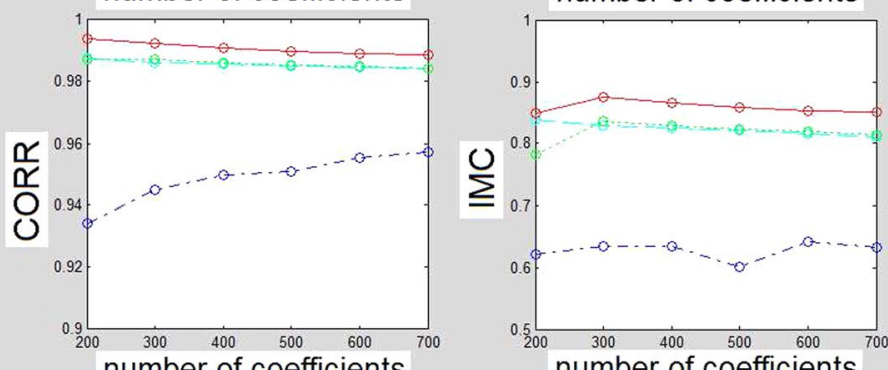

number of coefficients
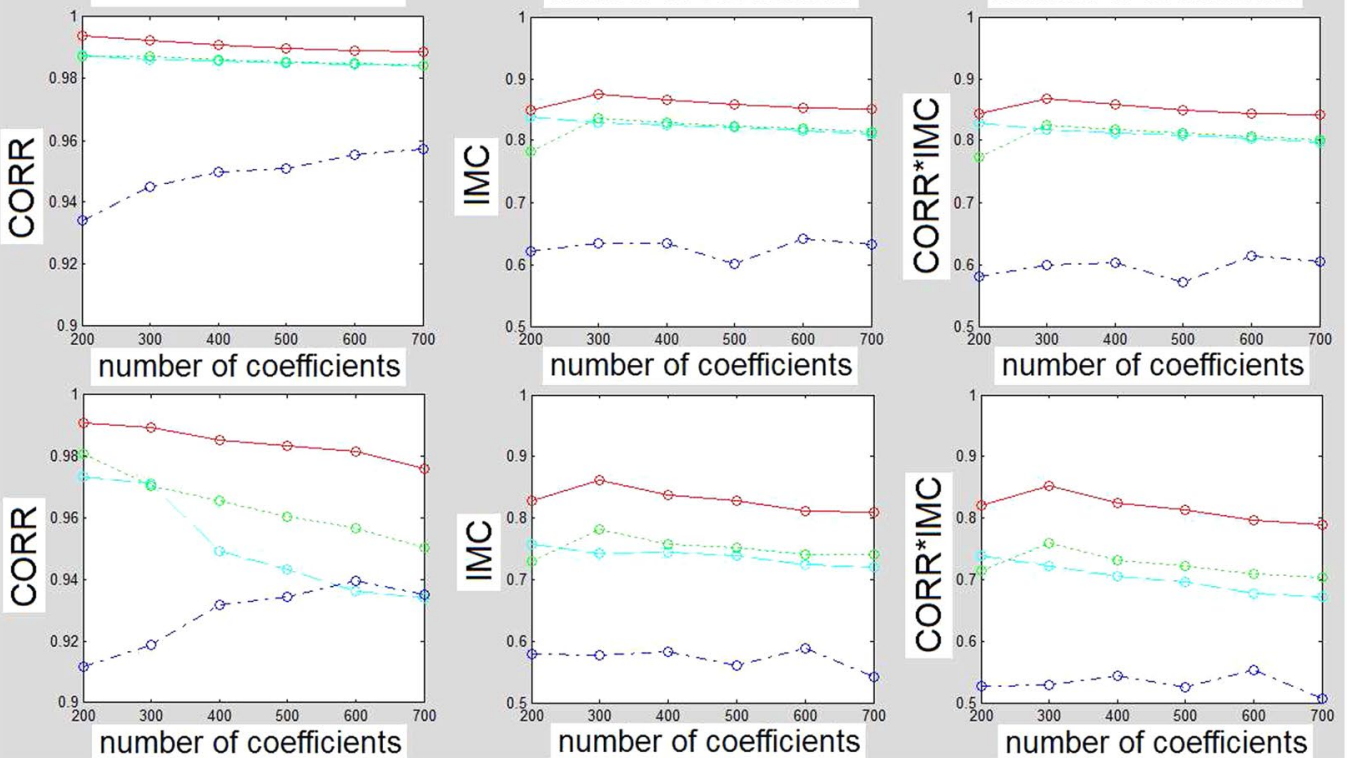

Fig. 7 The proposed objective assessment measure of spicule representation for mammographic ROIs (Fig. 5) with architectural distortions (a b) and normal breast tissue (c)

various transform-domain redundancies. Nonetheless, the concept of sparse approximation of texture directionality in the complex wavelet domain may be a useful tool at the stage of image preprocessing or directional structure extraction.

Acknowledgements This publication was funded by the National Science Centre (Poland) based on the decision DEC-2011/03/B/ ST7/03649.

Open Access This article is distributed under the terms of the Creative Commons Attribution 4.0 International License (http://creativeco mmons.org/licenses/by/4.0/), which permits unrestricted use, distribution, and reproduction in any medium, provided you give appropriate credit to the original author(s) and the source, provide a link to the Creative Commons license, and indicate if changes were made.

\section{References}

1. American College of Radiology (1998) Breast imaging reporting and data system (BI-RADS), 3rd edn. Atlas, Melbourne

2. Sampat MP, Markey MK, Bovik AC (2005) Computer-aided detection and diagnosis in mammography. In: Bovik AC (ed)
Handbook of image and video processing, 2nd edn. Academic, New York, pp 1195-1217

3. U.S. Food and Drug Administration (1998) Summary of safety and effectiveness data: R2 technologies, P970058

4. U.S. Food and Drug Administration (2002) Summary of safety and effectiveness data: CADx medical systems, PO10034

5. Vyborny CJ, Doi T, O'Shaughnessy KF, Romsdahl HM, Schneider AC, Stein AA (2000) Breast cancer: importance of spiculation in computer-aided detection. Radiology 215(3):703-707

6. Mallat S (2009) A wavelet tour of signal processing. Academic Press, New York

7. Candes EJ, Demanet L, Donoho DL, Ying L (2005) Fast discrete curvelet transforms. Multiscale Model Simul 5(3):861899

8. Candes EJ, Donoho DL (1999) Curvelets-a surprisingly effective nonadaptive representation for objects with edges. In: Schumaker LL et al (eds) Curves and surfaces. Vanderbilt University Press, Nashville

9. Do MN (2005) The contourlet transform: an efficient directional multiresolution image representation. IEEE Trans Image Process 14(12):2091-2106

10. Kingsbury NG (1999) Shift invariant properties of the dual-tree complex wavelet transform. Proc IEEE Conf Acoust Speech Signal Process 3:1221-1224

11. Kingsbury N (2001) Complex wavelets for shift invariant analysis and filtering of signals. J Appl Comput Harmonic Anal 10(3):234-253 
12. Sampat MP, Markey MK, Bovik AC (2006) Measurement and detection of spiculated lesions. IEEE Conf Image Anal Interpret 5747:105-109

13. Jasionowska M, Przelaskowski A, Jozwiak R (2010) Characteristics of architectural distortions in mammograms-extraction of texture orientation with Gabor filters. Computer vision and graphics, lecture notes in computer science, vol 6374, pp 420-430. Springer, Berlin

14. Adel M, Zuwala D, Rasigni M, Bourennane S (2006) Noise reduction on mammographic phantom images. Electron Lett Comput Vis Image Anal 5(4):64-74
15. Poularikas AD (2010) Transforms and applications handbook, 3rd edn. Taylor and Francis, New York

16. Yang G, Fang X, Jing M, Zhang S, Hou M (2010) Contourlet filter design based on Chebyshev best uniform approximation. EURASIP J Adv Sig Process 2010:398385-1-398385-10

17. University of South Florida, Digital Database for Screening Mammography (DDSM), Florida, USA. http://marathon.csee.usf.edu/ Mammography/Database.html

18. Haralick RM, Shanmugam K, Its'Hak D (1973) Textural features for image classification. IEEE Trans Syst Man Cybern SMC-3(6):610-621 